\title{
Weighted projections into closed subspaces
}

\author{
by \\ G. Corach, G. Fongi and A. Maestripieri (Buenos Aires)
}

\begin{abstract}
We study $A$-projections, i.e. operators on a Hilbert space $\mathcal{H}$ which act as projections when a seminorm is considered in $\mathcal{H}$. The $A$-projections were introduced by Mitra and Rao (1974) for finite-dimensional spaces. We relate this concept to the theory of compatibility between positive operators and closed subspaces of $\mathcal{H}$. We also study the relationship between weighted least squares problems and compatibility.
\end{abstract}

1. Introduction. In 1974, S. K. Mitra and C. R. Rao [21] introduced the notion of projection into a subspace with respect to a seminorm. More precisely, given a positive (semidefinite) matrix $A \in \mathbb{C}^{n \times n}$ and a subspace $\mathcal{S}$ of $\mathbb{C}^{n}$, a matrix $T \in \mathbb{C}^{n \times n}$ is called an $A$-projection into $\mathcal{S}$ if $R(T) \subseteq \mathcal{S}$ and

$$
\|y-T y\|_{A} \leq\|y-s\|_{A} \quad \text { for all } y \in \mathbb{C}^{n}, s \in \mathcal{S},
$$

where $\|z\|_{A}:=\langle A z, z\rangle^{1 / 2}=:\langle z, z\rangle_{A}^{1 / 2}$. Notice that an $A$-projection $T$ need not be an idempotent, but $A T^{2}=A T$. This notion is related to very general least squares problems and Mitra and Rao have found several applications in statistics, in particular in linear models (see also [24, 28, 29]).

In 1994, S. Hassi and K. Nordström [19] started the study of projections onto closed subspaces in Hilbert spaces, which are orthogonal with respect to an indefinite seminorm. Their paper suggested the notion of compatibility, proposed by G. Corach, A. Maestripieri and D. Stojanoff [8, 9, 10]. A closed subspace $\mathcal{S}$ of a Hilbert space $\mathcal{H}$ is said to be compatible with a positive (semidefinite bounded linear) operator $A$ on $\mathcal{H}$ if there exists a (bounded linear) projection $Q$ acting on $\mathcal{H}$ such that $\mathcal{S}$ is the image of $Q$ and $A Q=$ $Q^{*} A$. This equality means that $Q$ is selfadjoint with respect to the semi-inner product defined by $A$. The notion has several applications in generalized contractions [5, 26, 27], Krein space operators [19, 20], frame theory [2], least squares problems [7], signal processing [14, 15] and so on. It should be noticed that noncompatible pairs exist only if $\mathcal{H}$ has infinite dimension

2010 Mathematics Subject Classification: Primary 47A07; Secondary 47A58.

Key words and phrases: projections under seminorm, compatibility, weighted least squares problems. 
[10, 6.2]. Therefore, in order to study the relationship between Mitra-Rao's theory with the compatibility results, which is the main goal of this paper, it is necessary to extend that theory to the infinite-dimensional case.

Section 2 contains notations and preliminary results, in particular the well-known Douglas factorization theorem [13, 17]. Section 3 contains a short résumé of definitions and the main results of compatibility theory with no proof. In particular, if $(A, \mathcal{S})$ is compatible then a description of the set

$$
\mathcal{P}(A, \mathcal{S})=\left\{Q \in L(\mathcal{H}): Q^{2}=Q, R(Q)=\mathcal{S}, A Q=Q^{*} A\right\}
$$

is presented. Section 4 is devoted to developing the theory of $A$-projections in the context of infinite-dimensional Hilbert spaces. We only include proofs if they are not similar to those for finite-dimensional spaces provided by Mitra and Rao [21, 24]. The set $\Pi(A, \mathcal{S})=\{T \in L(\mathcal{H}): T$ is an $A$ projection into $\mathcal{S}\}$ is described and the precise relationship between $\mathcal{P}(A, \mathcal{S})$ and $\Pi(A, \mathcal{S})$ is presented, in the main result of the section, together with some minimality properties.

Section 5 deals with least squares problems. An operator $G \in L(\mathcal{H})$ is called an $A$-inverse of a closed range operator $B$ if for each $y \in \mathcal{H}, G y$ is an $A$-LSS of $B x=y$, i.e.

$$
\|B G y-y\|_{A} \leq\|B x-y\|_{A}, \quad x \in \mathcal{H} .
$$

We show that the existence of an $A$-inverse of an operator $B$ is equivalent to the compatibility of the pair $(A, R(B))$. Moreover the set of all $A$-inverses of $B$ is described. The second part of this section deals with restricted $A$ inverses of an operator $B: G \in L(\mathcal{H})$ is called an $A$-inverse of $B$ restricted to $\mathcal{M}$ if $R(G) \subseteq \mathcal{M}$ and

$$
\|B G y-y\|_{A} \leq\|B x-y\|_{A}, \quad \forall x \in \mathcal{M} .
$$

This notion, also due to Rao and Mitra [24, is completely described in terms of some compatibility conditions. In particular, there exists such a $G$ if and only if $(A, B(\mathcal{M}))$ is compatible. The final part deals with the least squares solution of an equation like

$$
B x=y
$$

where the vectors $x$ are measured with the seminorm \|\|$_{A_{1}}$ defined by $A_{1} \in$ $L(\mathcal{H})^{+}$and the vectors $y$ are measured with \|\|$_{A_{2}}$ for another $A_{2} \in L(\mathcal{H})^{+}$. Again, the situation is completely described by using certain compatibility conditions. Analogous problems have been considered in [7] and [18]. It should also be mentioned that L. Eldén [16] was the first to study this problem in finite dimensions.

2. Preliminaries. Throughout, $(\mathcal{H},\langle\rangle$,$) denotes a separable complex$ Hilbert space, $L(\mathcal{H})$ the algebra of bounded linear operators of $\mathcal{H}$, and $L(\mathcal{H})^{+}$ 
the cone of (semidefinite) positive operators, i.e. bounded linear operators satisfying $\langle A x, x\rangle \geq 0$ for all $x \in \mathcal{H}$. Also, $\mathcal{Q}$ denotes the subset of $L(\mathcal{H})$ of oblique projections, i.e. $\mathcal{Q}=\left\{Q \in L(\mathcal{H}): Q^{2}=Q\right\}$, and $\mathcal{P}$ the set of orthogonal projections, i.e. $\mathcal{P}=\left\{P \in L(\mathcal{H}): P^{2}=P=P^{*}\right\}$.

For every $A \in L(\mathcal{H}), R(A)$ denotes the range of $A$ and $N(A)$ its nullspace. Given two closed subspaces $\mathcal{M}$ and $\mathcal{N}$ of $\mathcal{H}, \mathcal{M} \dot{+} \mathcal{N}$ denotes the direct sum of $\mathcal{M}$ and $\mathcal{N}, \mathcal{M} \oplus \mathcal{N}$ the orthogonal sum and $\mathcal{M} \ominus \mathcal{N}=\mathcal{M} \cap(\mathcal{M} \cap \mathcal{N})^{\perp}$. If $\mathcal{M}+\mathcal{N}=\mathcal{H}$, denote by $P_{\mathcal{M} / / \mathcal{N}}$ the oblique projection with range $\mathcal{M}$ and nullspace $\mathcal{N}$; we write $P_{\mathcal{M}}=P_{\mathcal{M} / / \mathcal{M}^{\perp}}$.

Given a closed range operator $A, A^{\dagger}$ denotes the Moore-Penrose inverse of $A$, i.e. $A^{\dagger}$ is the unique solution of the system

$$
A X A=A, \quad X A X=X, \quad(A X)^{*}=A X, \quad(X A)^{*}=X A .
$$

Given a closed subspace $\mathcal{S}$ of $\mathcal{H}$, the projection $P_{\mathcal{S}}$ induces a matrix decomposition for each $A \in L(\mathcal{H})$ as follows: if $P=P_{\mathcal{S}}$ then $A \in L(\mathcal{H})$ can be written as

$$
A=\left(\begin{array}{ll}
a_{11} & a_{12} \\
a_{21} & a_{22}
\end{array}\right),
$$

where $a_{11}=\left.P A P\right|_{\mathcal{S}} \in L(\mathcal{S}), a_{12}=\left.P A(I-P)\right|_{\mathcal{S}^{\perp}} \in L\left(\mathcal{S}^{\perp}, \mathcal{S}\right), a_{21}=$ $\left.(I-P) A P\right|_{\mathcal{S}} \in L\left(\mathcal{S}, \mathcal{S}^{\perp}\right)$ and $a_{22}=\left.(I-P) A(I-P)\right|_{\mathcal{S}^{\perp}} \in L\left(\mathcal{S}^{\perp}\right)$. If $A \in L(\mathcal{H})^{+}$, then

$$
A=\left(\begin{array}{cc}
a & b \\
b^{*} & c
\end{array}\right)
$$

with $R(b) \subseteq R\left(a^{1 / 2}\right)$ (see [1]). Throughout this work, we will use the matrix representation of $A$ given by (1).

Given $A \in L(\mathcal{H})^{+}$, consider the following semi-inner product on $\mathcal{H}$ :

$$
\langle x, y\rangle_{A}=\langle A x, y\rangle, \quad x, y \in \mathcal{H} .
$$

The associated seminorm is given by

$$
\|x\|_{A}=\langle x, x\rangle_{A}^{1 / 2}=\left\|A^{1 / 2} x\right\|, \quad x \in \mathcal{H} .
$$

An operator $C \in L(\mathcal{H})$ is called $A$-selfadjoint if $\langle C x, y\rangle_{A}=\langle x, C y\rangle_{A}$ for all $x, y \in \mathcal{H}$, or equivalently $A C=C^{*} A$.

The following result, due to R. G. Douglas, characterizes the operator range inclusion (see [13, Theorem 1] and [17, Theorem 2.1] for the proof).

Theorem 2.1 (Douglas). Consider Hilbert spaces $\mathcal{H}, \mathcal{K}, \mathcal{G}$ and operators $A \in L(\mathcal{H}, \mathcal{G})$ and $B \in L(\mathcal{K}, \mathcal{G})$. The following conditions are equivalent:

(i) $R(B) \subseteq R(A)$,

(ii) $B B^{*} \leq \lambda A A^{*}$ for some $\lambda>0$,

(iii) the equation $A X=B$ has a solution in $L(\mathcal{K}, \mathcal{H})$. 
In this case, there exists a unique $D \in L(\mathcal{K}, \mathcal{H})$ such that $A D=B$ and $R(D) \subseteq \overline{R\left(A^{*}\right)}$; moreover, $\|D\|^{2}=\inf \left\{\lambda>0: B B^{*} \leq \lambda A A^{*}\right\}$ and $N(D)=$ $N(B)$. This solution is called the reduced solution of $A X=B$.

3. Compatibility. Given $A \in L(\mathcal{H})^{+}$and a closed subspace $\mathcal{S}$ of $\mathcal{H}$, consider the set

$$
\mathcal{P}(A, \mathcal{S})=\left\{Q \in \mathcal{Q}: R(Q)=\mathcal{S}, A Q=Q^{*} A\right\} .
$$

The pair $(A, \mathcal{S})$ is called compatible if $\mathcal{P}(A, \mathcal{S})$ is not empty, or equivalently, if there exists a projection $Q \in \mathcal{Q}$ with range $\mathcal{S}$ such that $A Q=Q^{*} A$.

The following proposition collects some results about compatibility that can be found in [9, 12].

Proposition 3.1. Consider $A \in L(\mathcal{H})^{+}$with matrix form given by equation (1), and a closed subspace $\mathcal{S}$ of $\mathcal{H}$.

(i) If the pair $(A, \mathcal{S})$ is compatible, then $\mathcal{S}+N(A)$ is closed.

(ii) If $A \in L(\mathcal{H})^{+}$has closed range and $\mathcal{S}+N(A)$ is closed, then $(A, \mathcal{S})$ is compatible.

(iii) The pair $(A, \mathcal{S})$ is compatible if and only if $\mathcal{H}=\mathcal{S}+A(\mathcal{S})^{\perp}$.

(iv) The pair $(A, \mathcal{S})$ is compatible if and only if $R(b) \subseteq R(a)$.

As a consequence of Douglas' theorem and item (iv) of the above proposition, we obtain the following characterization of the set $\mathcal{P}(A, \mathcal{S})$ (see [8] for details).

Corollary 3.2. If $(A, \mathcal{S})$ is compatible, then

$$
\mathcal{P}(A, \mathcal{S})=\left\{\left(\begin{array}{ll}
1 & x \\
0 & 0
\end{array}\right): x \in L\left(\mathcal{S}^{\perp}, \mathcal{S}\right) \text { and } a x=b\right\} .
$$

If the pair $(A, \mathcal{S})$ is compatible, there is a distinguished element $P_{A, \mathcal{S}} \in$ $\mathcal{P}(A, \mathcal{S})$, namely the unique projection onto $\mathcal{S}$ with kernel $A(\mathcal{S})^{\perp} \ominus \mathcal{N}$, where $\mathcal{N}=A(\mathcal{S})^{\perp} \cap \mathcal{S}=N(A) \cap \mathcal{S}$. By [10, Proposition 4.1], $P_{A, \mathcal{S}}=P_{A, \mathcal{S} \ominus \mathcal{N}}$ $+P_{\mathcal{N}}$ and $P_{A, \mathcal{S} \ominus \mathcal{N}}=P_{\mathcal{S} \ominus \mathcal{N} / / A(\mathcal{S})^{\perp}}$. Then the matrix decomposition of $P_{A, \mathcal{S}}$ induced by $P_{\mathcal{S}}$ is given by

$$
P_{A, \mathcal{S}}=\left(\begin{array}{ll}
1 & d \\
0 & 0
\end{array}\right)
$$

where $d \in L\left(\mathcal{S}^{\perp}, \mathcal{S}\right)$ is the reduced solution of $a x=b$.

It is easy to see that the pair $(A, \mathcal{S})$ is compatible if and only if the pair $(A, \mathcal{S} \ominus \mathcal{N})$ is compatible.

4. Weighted projections. Along this work $A$ is a positive bounded operator, i.e. $A \in L(\mathcal{H})^{+}$, and $\mathcal{S}$ is a closed subspace of $\mathcal{H}$. 
The following definition is due to Mitra and Rao [21] for operators acting on finite-dimensional Hilbert spaces.

Definition 4.1. An operator $T \in L(\mathcal{H})$ is called an $A$-projection into $\mathcal{S}$ if $R(T) \subseteq \mathcal{S}$ and

$$
\|y-T y\|_{A} \leq\|y-s\|_{A} \quad \text { for all } y \in \mathcal{H} \text { and all } s \in \mathcal{S} .
$$

$T$ is called an $A$-projection if $T$ is an $A$-projection into $\overline{R(T)}$.

An $A$-projection into $\mathcal{S}$ is also called an $A$-weighted least squares process (see [6, 25]).

REMARK 4.2. It is not difficult to see that inequality (2) alone does not imply the boundedness of $T$. Indeed, if $A$ has infinite-dimensional nullspace then it is enought to consider $T=T_{1} P_{N(A)}+P_{\overline{R(A)}}$, with $T_{1}: N(A) \rightarrow N(A)$ unbounded. Similarly, it can be proved that the range of an $A$-projection is not necessarily closed.

Definition 4.3. The operator $T \in L(\mathcal{H})$ is an $A$-idempotent if $A T^{2}$ $=A T$.

Observe that the definition of $A$-idempotent only depends on $N(A)$ in the sense that if $A, B \in L(\mathcal{H})$ are such that $N(A)=N(B)$ then $T$ is $A$ idempotent if and only if $T$ is $B$-idempotent.

The next two propositions generalize some of the results in [21]. The proofs for infinite-dimensional Hilbert spaces follow essentially the same steps.

Proposition 4.4. Let $T \in L(\mathcal{H})$. The following statements are equivalent:

(i) $T$ is an A-projection,

(ii) $T^{*} A T=A T$,

(iii) $A T=T^{*} A$ and $A T^{2}=A T$; or equivalently, $T$ is $A$-selfadjoint and also A-idempotent.

Proof. See [21, Lemmas 2.1 and 2.2].

Proposition 4.5. Consider $T \in L(\mathcal{H})$ such that $R(T) \subseteq \mathcal{S}$. The following conditions are equivalent:

(i) $T$ is an $A$-projection into $\mathcal{S}$,

(ii) $A T=T^{*} A$ and $A T P_{\mathcal{S}}=A P_{\mathcal{S}}$,

(iii) $P_{\mathcal{S}} A T=P_{\mathcal{S}} A$.

Proof. (i) $\Rightarrow$ (ii): Let $T$ be an $A$-projection into $\mathcal{S}$. In particular $T$ is an $A$-projection. Then by Proposition 4.4, $A T=T^{*} A$. On the other hand, for each $y \in \mathcal{H}$ we have $\|y-T y\|_{A}=\left\|A^{1 / 2} y-A^{1 / 2} T y\right\| \leq\|y-s\|_{A}$ for all $s \in \mathcal{S}$. 
In particular, if $x \in \mathcal{H}$ then $\left\|A^{1 / 2} P_{\mathcal{S}} x-A^{1 / 2} T P_{\mathcal{S}} x\right\| \leq\left\|P_{\mathcal{S}} x-s\right\|_{A}$ for all $s \in \mathcal{S}$. Therefore, $A^{1 / 2} P_{\mathcal{S}}=A^{1 / 2} T P_{\mathcal{S}}$, so that $A P_{\mathcal{S}}=A T P_{\mathcal{S}}$.

(ii) $\Rightarrow$ (iii): If $A T=T^{*} A$ and $A T P_{\mathcal{S}}=A P_{\mathcal{S}}$ then $P_{\mathcal{S}} A=P_{\mathcal{S}} T^{*} A=$ $P_{\mathcal{S}} A T$, so that $P_{\mathcal{S}} A=P_{\mathcal{S}} A T$.

(iii) $\Rightarrow\left(\right.$ i): Since $P_{\mathcal{S}} A T=P_{\mathcal{S}} A$, we see that $T^{*} A P_{\mathcal{S}}=A P_{\mathcal{S}}$ and so $T^{*} A T$ $=A T=T^{*} A$ because $R(T) \subseteq \mathcal{S}$. Therefore, by Proposition 4.4. $T$ is an $A$-projection into $\overline{R(T)}$, therefore $\|y-T y\|_{A} \leq\|y-T x\|_{A}$ for all $x, y \in \mathcal{H}$. It remains to prove that $\|y-T y\|_{A} \leq\left\|y-P_{\mathcal{S}} x\right\|_{A}$ for all $x, y \in \mathcal{H}$. Since $A T P_{\mathcal{S}}=A P_{\mathcal{S}}$, we have $A^{1 / 2} T P_{\mathcal{S}}=A^{1 / 2} P_{\mathcal{S}}$, so $\|y-T y\|_{A} \leq\left\|y-T P_{\mathcal{S}} x\right\|_{A}=$ $\left\|y-P_{\mathcal{S}} x\right\|_{A}$ for all $x, y \in \mathcal{H}$.

Remark 4.6. By Propositions 4.4 and 4.5 , given $T \in L(\mathcal{H})$ such that $R(T) \subseteq \mathcal{S}$ we can see that $T$ is an $A$-projection into $\mathcal{S}$ if and only if $T$ is an $A$-projection and $A T P_{\mathcal{S}}=A P_{\mathcal{S}}$.

Lemma 4.7. If $T \in L(\mathcal{H})$ is an A-idempotent (resp. A-projection) then $I-T$ is an A-idempotent (resp. A-projection).

Proof. If $T \in L(\mathcal{H})$ is an $A$-idempotent, then $A(I-T)^{2}=A\left(I-2 T+T^{2}\right)$ $=A(I-T)$, i.e. $I-T$ is an $A$-idempotent. If $T$ is an $A$-projection then, by Proposition 4.4. $T$ is $A$-idempotent and $A$-selfadjoint. Hence $I-T$ is $A$ idempotent and $A(I-T)=A-T^{*} A=(I-T)^{*} A$. Again by Proposition 4.4. $I-T$ is an $A$-projection.

The following result characterizes $A$-projections in terms of oblique projections.

Lemma 4.8. Let $T \in L(\mathcal{H})$. Then $T$ is an A-projection if and only if $P_{\overline{R(A)}} T \in \mathcal{Q}$ and it is A-selfadjoint.

Proof. Let $T$ be an $A$-projection and denote $P=P_{\overline{R(A)}}$. By Proposition 4.4, we find that $A T=T^{*} A$ and $A T=A T^{2}$, hence $(P T)^{2}=A^{\dagger} A T P T$ $=A^{\dagger} T^{*} A P T=A^{\dagger} T^{*} A T=A^{\dagger} A T=P T$ and $(P T)^{*} A=T^{*} P A=T^{*} A=$ $A T=A P T$. Conversely, if $P T \in \mathcal{Q}$ and it is $A$-selfadjoint then $A T=$ $A P T=(P T)^{*} A=T^{*} A$ so that $T$ is $A$-selfadjoint. Also, $A T^{2}=A P T^{2}=$ $(P T)^{*} A T=(P T)^{*} A P T=A(P T)^{2}=A P T=A T$ so that $T$ is $A$-idempotent. By Proposition 4.4, $T$ is an $A$-projection.

The next result shows that $A$-projections behave like orthogonal projections, under the seminorm induced by $A$, in the sense that for an $A$ idempotent, the condition of being $A$-selfadjoint is equivalent to being an $A$-contraction, or $A$-positive. For $A$-contractions see for example [5] and [26].

Proposition 4.9. Let $T \in L(\mathcal{H})$ be an A-idempotent. Then the following statements are equivalent: 
(i) $T$ is A-selfadjoint (so that $T$ is an A-projection),

(ii) $R(I-T) \subseteq R(A T)^{\perp}$,

(iii) $T$ is an $A$-contraction, i.e. $T^{*} A T \leq A$.

Proof. (i) $\Rightarrow\left(\right.$ ii): Suppose that $A T=T^{*} A$. Consider $y \in R(I-T)$ and $z \in \mathcal{H}$ such that $y=z-T z$. Then, for $x \in \mathcal{H}$,

$$
\langle A T x, y\rangle=\langle x, A T y\rangle=\langle x, A T(z-T z)\rangle=0,
$$

because $A T^{2}=A T$. Therefore, $y \in R(A T)^{\perp}$.

(ii) $\Rightarrow($ iii): For $x, y \in \mathcal{H}$,

$$
\langle A T x, y\rangle=\langle A T x, T y+(I-T) y\rangle=\langle A T x, T y\rangle=\left\langle T^{*} A T x, y\right\rangle
$$

because $R(I-T) \subseteq R(A T)^{\perp}$. Therefore, $A T=T^{*} A T=T^{*} A$ and $T$ is $A$-selfadjoint. Then $T$ is an $A$-projection. Also, by Lemma 4.7, $E=I-T$ is an $A$-projection so that $A E=A E^{2}=E^{*} A E \in L(\mathcal{H})^{+}$. Therefore, $A=$ $A(T+E)=T^{*} A T+E^{*} A E \geq T^{*} A T$.

(iii) $\Rightarrow\left(\right.$ i): Since $T^{*} A T \leq \bar{A}$, by Douglas' theorem, the equation $A^{1 / 2} X=$ $T^{*} A^{1 / 2}$ admits a solution. Let $D$ be the reduced solution of $A^{1 / 2} X=T^{*} A^{1 / 2}$, i.e. $D$ satisfies $A^{1 / 2} D=T^{*} A^{1 / 2}$ and $R(D) \subseteq \overline{R(A)}$. Then observe that

$$
A^{1 / 2} D^{2}=\left(T^{*}\right)^{2} A^{1 / 2}=T^{*} A^{1 / 2},
$$

because $T$ is an $A$-idempotent. Also, $R\left(D^{2}\right) \subseteq R(D) \subseteq \overline{R(A)}$. Therefore $D^{2}$ is a reduced solution of $A^{1 / 2} X=T^{*} A^{1 / 2}$ as well so that $D^{2}=D$ by the uniqueness of the reduced solution. On the other hand, by Douglas' theorem, $\|D\|^{2}=\inf \left\{\lambda: T^{*} A T \leq \lambda A\right\} \leq 1$, because $T^{*} A T \leq A$. Since $D^{2}=D$ and $\|D\| \leq 1$, automatically $D^{*}=D$, so that $T^{*} A=A^{1 / 2} D A^{1 / 2}$ is selfadjoint, i.e. $T^{*} A=A T$.

Corollary 4.10. Let $T \in L(\mathcal{H})$ be an A-idempotent. The following statements are equivalent:

(i) $T$ is an A-projection,

(ii) $\|T\|_{A}=1$,

(iii) $\langle\text { Tx, } x\rangle_{A} \geq 0$ for all $x \in \mathcal{H}$, i.e. $T$ is $A$-positive.

Proof. (i) $\Rightarrow$ (ii): Since $A$ is an $A$-projection, by Proposition 4.9, we have $T^{*} A T \leq A$. Then, for $x \in \mathcal{H}$,

$$
\|T x\|_{A}^{2}=\langle A T x, T x\rangle=\left\langle T^{*} A T x, x\right\rangle \leq\langle A x, x\rangle=\|x\|_{A}^{2},
$$

so that $\|T\|_{A} \leq 1$. Also,

$$
\|T(T x)\|_{A}=\left\|A^{1 / 2} T^{2} x\right\|=\left\|A^{1 / 2} T x\right\|=\|T x\|_{A},
$$

because $T$ is $A$-idempotent. Therefore $\|T\|_{A}=1$.

(ii) $\Rightarrow$ (iii): Consider $T \in L(\mathcal{H})$ such that $A T=A T^{2}$ and $\|T\|_{A}=1$. Observe that

$$
\left\langle T^{*} A T x, x\right\rangle=\langle A T x, T x\rangle=\|T x\|_{A}^{2} \leq\|x\|_{A}^{2}=\langle A x, x\rangle,
$$


so that $T^{*} A T \leq A$, and by Proposition $4.9, T$ is an $A$-projection. By Proposition 4.4 , it follows that $A T=T^{*} A T \in L(\mathcal{H})^{+}$.

(iii) $\Rightarrow(\mathrm{i})$ : Since $A T \in L(\mathcal{H})^{+}$, we have $A T=T^{*} A$. Also, $T$ is $A$-idempotent so that, by Proposition $4.4, T$ is an $A$-projection.

In the following paragraphs we study conditions for the existence of $A$ projections into $\mathcal{S}$ and we characterize the set of these projections.

Define

$$
\begin{aligned}
\Pi(A, \mathcal{S}) & =\{T \in L(\mathcal{H}): T \text { is an } A \text {-projection into } \mathcal{S}\}, \\
\Pi(A) & =\{T \in L(\mathcal{H}): T \text { is an } A \text {-projection }\} .
\end{aligned}
$$

By Proposition 4.5, it follows that

$$
\Pi(A, \mathcal{S})=\left\{T \in L(\mathcal{H}): R(T) \subseteq \mathcal{S}, A T=T^{*} A, A T P_{\mathcal{S}}=A P_{\mathcal{S}}\right\}
$$

and

$$
\Pi(A)=\left\{T \in L(\mathcal{H}): A T=T^{*} A, A T^{2}=A T\right\} .
$$

In particular if $A=I$, then $\Pi(I, \mathcal{S})=\left\{P_{\mathcal{S}}\right\}$ and $\Pi(I)=\mathcal{P}$.

The next result gives a characterization of $A$-projections into $\mathcal{S}$ in terms of the matrix decomposition induced by $P_{\mathcal{S}}$. Recall that $A=\left(\begin{array}{cc}a & b \\ b^{*} & c\end{array}\right)$ is the matrix representation of $A$, as in (1).

Proposition 4.11. $\Pi(A, \mathcal{S})=\left\{T \in L(\mathcal{H}): T=\left(\begin{array}{ll}x & y \\ 0 & 0\end{array}\right), a x=a\right.$, $a y=b\}$.

Proof. By equation (3), $T \in \Pi(A, \mathcal{S})$ if and only if $R(T) \subseteq \mathcal{S}, T^{*} A=$ $A T$ and $A T P_{\mathcal{S}}=A P_{\mathcal{S}}$. Observe that $R(T) \subseteq \mathcal{S}$ if and only if the matrix representation of $T$ induced by $P_{\mathcal{S}}$ is $T=\left(\begin{array}{cc}x & y \\ 0 & 0\end{array}\right)$. In this case, $A T=T^{*} A$ if and only if $a x=x^{*} a, a y=x^{*} b$ and $b^{*} y=y^{*} b$. Also, $A T P_{\mathcal{S}}=A P_{\mathcal{S}}$ is equivalent to $a x=a$ and $b^{*} x=b^{*}$. Then $T \in \Pi(A, \mathcal{S})$ if and only if

$$
a x=x^{*} a, \quad a y=x^{*} b, \quad b^{*} y=y^{*} b, \quad a x=a, \quad b^{*} x=b^{*} .
$$

It is not difficult to see that (4) is equivalent to $a x=a$ and $a y=b$.

Corollary 4.12. If the pair $(A, \mathcal{S})$ is compatible, then

$$
\mathcal{P}(A, \mathcal{S}) \subseteq \Pi(A, \mathcal{S}) .
$$

Proof. This follows from Corollary 3.2 and Proposition 4.11.

Applying item (iii) of Proposition 4.5, we obtain the following

Corollary 4.13. $\Pi(A, \mathcal{S})=\{T \in L(\mathcal{H}): R(T) \subseteq \mathcal{S}$ and $T$ is a solution of the equation $\left.P_{\mathcal{S}} A X=P_{\mathcal{S}} A\right\}$.

The next result shows the relationship between the compatibility of the pair $(A, \mathcal{S})$ and the existence of $A$-projections into $\mathcal{S}$.

Proposition 4.14. The pair $(A, \mathcal{S})$ is compatible if and only if there exists an A-projection into $\mathcal{S}$. 
Proof. By Proposition 4.11, the set $\Pi(A, \mathcal{S})$ is not empty if and only if the equation $a y=b$ admits a solution (observe that $a x=a$ always admits a solution). By Douglas' theorem this is equivalent to the condition $R(b) \subseteq$ $R(a)$, or equivalently by Proposition 3.1 , the pair $(A, \mathcal{S})$ is compatible.

REMARK 4.15. By the above proposition, $\Pi(A, \mathcal{S}) \neq \emptyset$ if and only if $\mathcal{P}(A, \mathcal{S}) \neq \emptyset$.

Recall that $\mathcal{N}=\mathcal{S} \cap A(\mathcal{S})^{\perp}=\mathcal{S} \cap N(A)$.

Proposition 4.16. Let $T \in L(\mathcal{H})$ with $R(T) \subseteq \mathcal{S}$. Then $T$ is an $A$ projection into $\mathcal{S}$ if and only if $(A, \mathcal{S})$ is compatible and $P_{\mathcal{S} \ominus \mathcal{N}} T=P_{A, \mathcal{S} \ominus \mathcal{N}}$.

Proof. Suppose $T$ is an $A$-projection into $\mathcal{S}$. Let $Q=P_{\mathcal{S} \ominus \mathcal{N}} T$. Then $R(Q) \subseteq \mathcal{S}$ and $A T=A Q$. Since $T$ is an $A$-projection, $A Q=A T=T^{*} A=$ $Q^{*} A$, so $Q$ is $A$-selfadjoint. Moreover $A Q P_{\mathcal{S}}=A T P_{\mathcal{S}}=A P_{\mathcal{S}}$, hence $Q$ is an $A$-projection into $\mathcal{S}$. Therefore $A Q^{2}=A Q$ so that $R\left(Q^{2}-Q\right) \subseteq$ $N(A) \cap(\mathcal{S} \ominus \mathcal{N})=\mathcal{N} \cap \mathcal{N}^{\perp}=\{0\}$, or equivalently $Q^{2}=Q$. Moreover, from $A Q P_{\mathcal{S}}=A P_{\mathcal{S}}$ and $A Q=Q^{*} A$ it follows that $A Q P_{\mathcal{S} \ominus \mathcal{N}}=Q^{*} A P_{\mathcal{S} \ominus \mathcal{N}}=$ $Q^{*} A P_{\mathcal{S}}=A Q P_{\mathcal{S}}=A P_{\mathcal{S}}=A P_{\mathcal{S} \ominus \mathcal{N}}$. Therefore $A\left(Q P_{\mathcal{S} \ominus \mathcal{N}}-P_{\mathcal{S} \ominus \mathcal{N}}\right)=0$. Also $R\left(Q P_{\mathcal{S} \ominus \mathcal{N}}-P_{\mathcal{S} \ominus \mathcal{N}}\right) \subseteq \mathcal{S} \ominus \mathcal{N}$. Hence $R\left(Q P_{\mathcal{S} \ominus \mathcal{N}}-P_{\mathcal{S} \ominus \mathcal{N}}\right) \subseteq N(A) \cap$ $(\mathcal{S} \ominus \mathcal{N})=\{0\}$, so that $Q P_{\mathcal{S} \ominus \mathcal{N}}=P_{\mathcal{S} \ominus \mathcal{N}}$ and then $\mathcal{S} \ominus \mathcal{N} \subseteq R(Q)$. Therefore $R(Q)=\mathcal{S} \ominus \mathcal{N}$. Since $A Q=Q^{*} A, Q^{2}=Q$ and $R(Q)=\mathcal{S} \ominus \mathcal{N}$, it follows that $Q=P_{A, \mathcal{S} \ominus \mathcal{N}}$. Then $(A, \mathcal{S} \ominus \mathcal{N})$ is compatible, so that $(A, \mathcal{S})$ is compatible (see Section 3).

Conversely, if $(A, \mathcal{S})$ is compatible and $P_{\mathcal{S} \ominus \mathcal{N}} T=P_{A, \mathcal{S} \ominus \mathcal{N}}$, then $T=$ $P_{A, \mathcal{S} \ominus \mathcal{N}}+P_{\mathcal{N}} T$, so that $A T=T^{*} A$ and $A T P_{\mathcal{S}}=A P_{A, \mathcal{S} \ominus \mathcal{N}} P_{\mathcal{S}}=A P_{\mathcal{S} \ominus \mathcal{N}}=$ $A P_{\mathcal{S}}$. Then $T$ is an $A$-projection into $\mathcal{S}$.

The following result shows that $\Pi(A, \mathcal{S})$ is an affine manifold.

Proposition 4.17. If the pair $(A, \mathcal{S})$ is compatible, then

$$
\Pi(A, \mathcal{S})=P_{A, \mathcal{S}}+L(\mathcal{H}, \mathcal{N}) .
$$

Proof. Let $T \in \Pi(A, \mathcal{S})$. Then by Proposition 4.16, it follows that $T=$ $P_{A, \mathcal{S} \ominus \mathcal{N}}+P_{\mathcal{N}} T=P_{A, \mathcal{S}}+P_{\mathcal{N}}(T-I) \in P_{A, \mathcal{S}}+L(\mathcal{H}, \mathcal{N})$ (see Section 3).

Conversely, if $T=P_{A, \mathcal{S}}+W$ with $W \in L(\mathcal{H}, \mathcal{N})$, then $P_{\mathcal{S} \ominus \mathcal{N}} T=P_{A, \mathcal{S} \ominus \mathcal{N}}$. By Proposition 4.16, $T$ is an $A$-projection into $\mathcal{S}$.

Remark 4.18. Given $T \in \Pi(A, \mathcal{S})$, observe that $A T=A P_{A, \mathcal{S}}$ since $\mathcal{N} \subseteq N(A)$. Hence $A(R(T))=R(A T)=A(\mathcal{S})$.

A natural question is whether $\mathcal{P}(A, \mathcal{S})$ equals $\Pi(A, \mathcal{S})$. We prove now that this happens if and only if $\mathcal{P}(A, \mathcal{S})$ and/or $\Pi(A, \mathcal{S})$ has cardinal 1.

TheOREM 4.19. Suppose that the pair $(A, \mathcal{S})$ is compatible. Then the following statements are equivalent: 
(i) $\mathcal{P}(A, \mathcal{S})=\Pi(A, \mathcal{S})$,

(ii) $\mathcal{N}=\{0\}$,

(iii) $\operatorname{card}(\Pi(A, \mathcal{S}))=1$,

(iv) $\operatorname{card}(\mathcal{P}(A, \mathcal{S}))=1$.

Proof. (i) $\Rightarrow$ (ii): Suppose $\mathcal{N} \neq\{0\}$ and consider $T=P_{A, \mathcal{S}}+P_{\mathcal{N}}$. Then, by the previous theorem, $T \in \Pi(A, \mathcal{S})$. But it is not difficult to see that $T^{2} \neq T$, whence $T \notin \mathcal{P}(A, \mathcal{S})$.

(ii) $\Rightarrow$ (iii): If $\mathcal{N}=\{0\}$, by the previous theorem $\Pi(A, \mathcal{S})=\left\{P_{A, \mathcal{S}}\right\}$.

(iii) $\Rightarrow$ (iv): Apply Corollary 4.12 and Remark 4.15 .

(iv) $\Rightarrow($ i): If $\operatorname{card}(\mathcal{P}(A, \mathcal{S}))=1$, then by [8, Theorem 3.5], $\mathcal{N}=\{0\}$ and $\mathcal{P}(A, \mathcal{S})=\left\{P_{A, \mathcal{S}}\right\}$. Hence, by the previous result, $\Pi(A, \mathcal{S})=\left\{P_{A, \mathcal{S}}\right\}=$ $\mathcal{P}(A, \mathcal{S})$.

Corollary 4.20. If $A$ is invertible, then $\Pi(A, \mathcal{S})=\mathcal{P}(A, \mathcal{S})=\left\{P_{A, \mathcal{S}}\right\}$.

REMARK 4.21. Under the hypothesis of the above corollary, $P_{A, \mathcal{S}}$ can be computed as

$$
P_{A, \mathcal{S}}=A^{-1 / 2} P_{\overline{A^{1 / 2}(\mathcal{S})}} A^{1 / 2}
$$

(see [29, Section 3] or, more generally, [11, Proposition 3.3]).

Some minimality properties of $P_{A, \mathcal{S}}$ with respect to $\mathcal{P}(A, \mathcal{S})$ are proved in [8, Theorem 3.5] and [9, Theorem 3.2]. The next result extends these properties to the set $\Pi(A, \mathcal{S})$.

Proposition 4.22. Suppose that the pair $(A, \mathcal{S})$ is compatible. Then

(i) $\left\|P_{A, \mathcal{S}}\right\|=\min \{\|T\|: T \in \Pi(A, \mathcal{S})\}$.

(ii) $\left\|\left(I-P_{A, \mathcal{S}}\right) x\right\| \leq\|(I-T) x\|$ for all $x \in \mathcal{H}$ and every $T \in \Pi(A, \mathcal{S})$.

Proof. (i) Consider $T \in \Pi(A, \mathcal{S})$. Then, by Proposition 4.16, $T=$ $P_{A, \mathcal{S} \ominus \mathcal{N}}+W$ for some $W \in L(\mathcal{H}, \mathcal{N})$. So

$$
\|T x\|^{2}=\left\|P_{A, \mathcal{S} \ominus \mathcal{N}} x\right\|^{2}+\|W x\|^{2} \geq\left\|P_{A, \mathcal{S} \ominus \mathcal{N}} x\right\|^{2} \quad \text { for all } x \in \mathcal{H} .
$$

Therefore, $\|T\| \geq\left\|P_{A, \mathcal{S} \ominus \mathcal{N}}\right\|$. Finally, observe that

$$
\begin{aligned}
\left\|P_{A, \mathcal{S}}\right\|^{2} & =\left\|P_{A, \mathcal{S}}\left(P_{A, \mathcal{S}}\right)^{*}\right\|=\left\|P_{\mathcal{N}}+P_{A, \mathcal{S} \ominus \mathcal{N}}\left(P_{A, \mathcal{S} \ominus \mathcal{N}}\right)^{*}\right\| \\
& =\max \left\{\left\|P_{\mathcal{N}}\right\|,\left\|P_{A, \mathcal{S} \ominus \mathcal{N}}\right\|\right\}=\left\|P_{A, \mathcal{S} \ominus \mathcal{N}}\right\|^{2},
\end{aligned}
$$

because $P_{A, \mathcal{S}}=P_{A, \mathcal{S} \ominus \mathcal{N}}+P_{\mathcal{N}}, P_{A, \mathcal{S} \ominus \mathcal{N}} P_{\mathcal{N}}=0=P_{\mathcal{N}}\left(P_{A, \mathcal{S} \ominus \mathcal{N}}\right)^{*}$ and $P_{\mathcal{N}} P_{A, \mathcal{S} \ominus \mathcal{N}}=0=\left(P_{A, \mathcal{S} \ominus \mathcal{N}}\right)^{*} P_{\mathcal{N}}$.

(ii) Consider $T \in \Pi(A, \mathcal{S})$. By Proposition 4.17, $T=P_{A, \mathcal{S}}+W$ for some $W \in L(\mathcal{H}, \mathcal{N})$. Observe that

$\|(I-T) x\|^{2}=\left\|\left(I-P_{A, \mathcal{S}}\right) x\right\|^{2}+\|W x\|^{2} \leq\left\|\left(I-P_{A, \mathcal{S}}\right) x\right\|^{2} \quad$ for all $x \in \mathcal{H}$, because $R\left(I-P_{A, \mathcal{S}}\right)=N\left(P_{A, \mathcal{S}}\right)=A(\mathcal{S})^{\perp} \ominus \mathcal{N} \subseteq \mathcal{N}^{\perp}$.

As an application, we characterize the abstract splines in terms of weighted projections. The theory of abstract splines is due to Atteia [3]. The reader 
is referred to [10] for some relationships between the notion of compatibility and abstract splines in Hilbert spaces.

Given $C \in L(\mathcal{H})$, a closed subspace $\mathcal{S}$ of $\mathcal{H}$ and $x \in \mathcal{H}$, an abstract spline or a $(C, \mathcal{S})$-spline interpolant to $x$ is any element of the set

$$
\operatorname{sp}(C, \mathcal{S}, x)=\left\{y \in x+\mathcal{S}:\|C y\|=\min _{s \in \mathcal{S}}\|C(x+s)\|\right\} .
$$

If $A=C^{*} C \in L(\mathcal{H})^{+}$, observe that $\|y\|_{A}=\|C y\|$ for $y \in \mathcal{H}$. Then

$$
\operatorname{sp}(C, \mathcal{S}, x)=\left\{y \in x+\mathcal{S}:\|y\|_{A}=d_{A}(x, \mathcal{S})\right\},
$$

where $d_{A}(x, \mathcal{S})=\inf _{s \in \mathcal{S}}\|x+s\|_{A}$.

The next proposition contains some results on splines, the proofs can be found in [10].

Proposition 4.23. Given $C \in L(\mathcal{H})$, set $A=C^{*} C$. Then

(i) $\operatorname{sp}(C, \mathcal{S}, x)=(x+\mathcal{S}) \cap A(\mathcal{S})^{\perp}$ for $x \in \mathcal{H}$.

(ii) $\operatorname{sp}(C, \mathcal{S}, x) \neq 0$ for every $x \in \mathcal{H}$ if and only if the pair $(A, \mathcal{S})$ is compatible.

(iii) If $(A, \mathcal{S})$ is compatible and $x \in \mathcal{H} \backslash \mathcal{S}$, then $\operatorname{sp}(C, \mathcal{S}, x)=\{(I-Q) x$ : $Q \in \mathcal{P}(A, \mathcal{S})\}$.

Proposition 4.24. Consider $C \in L(\mathcal{H})$ and suppose that $(A, \mathcal{S})$ is compatible, where $A=C^{*} C$. For every nonzero $x \in \mathcal{H}$,

$$
\operatorname{sp}(C, \mathcal{S}, x)=\{(I-T) x: T \in \Pi(A, \mathcal{S})\} .
$$

Proof. Consider $y \in \operatorname{sp}(C, \mathcal{S}, x)$. By Proposition 4.23(i) there exists $s \in \mathcal{S}$ such that $x=s+y$ and $y=x-s \in A(\mathcal{S})^{\perp}$. We are looking for $T \in \Pi(A, \mathcal{S})$ such that $(I-T) x=y$, or equivalently $T x=s$. Note that $y_{1}=\left(I-P_{A, \mathcal{S}}\right) x \in$ $N\left(P_{A, \mathcal{S}}\right) \subseteq A(\mathcal{S})^{\perp}$. Then

$$
y_{1}-y=\left(I-P_{A, \mathcal{S}}\right) x-(x-s)=s-P_{A, \mathcal{S}} x \in \mathcal{S} \cap A(\mathcal{S})^{\perp}=\mathcal{N} .
$$

Therefore, since $x \neq 0$, we can consider $W \in L(\mathcal{H}, \mathcal{N})$ sucht that $W x=$ $s-P_{A, \mathcal{S}} x$. By Proposition 4.17 it follows that $T=P_{A, \mathcal{S}}+W \in \Pi(A, \mathcal{S})$; moreover $T x=s$.

Conversely, let $T \in \Pi(A, \mathcal{S})$. Then $(I-T) x \in(x+\mathcal{S}) \cap R(I-T)$. But, by Proposition 4.9 and Remark $4.18, R(I-T) \subseteq R(A T)^{\perp}=A(\mathcal{S})^{\perp}$. Therefore

$$
(I-T) x \in(x+\mathcal{S}) \cap A(\mathcal{S})^{\perp}=\operatorname{sp}(C, \mathcal{S}, x),
$$

by the above proposition.

Observe that, by Proposition 4.23(i), $\operatorname{sp}(C, \mathcal{S}, 0)=\mathcal{N}$.

5. Weighted inverses. Throughout this section, $A \in L(\mathcal{H})^{+}$and $B \in$ $L(\mathcal{H})$ is a closed range operator. 
Definition 5.1. Given $y \in \mathcal{H}, x_{0} \in \mathcal{H}$ is an $A$-least squares solution or an $A-L S S$ of $B x=y$ if

$$
\left\|y-B x_{0}\right\|_{A} \leq\|y-B x\|_{A}, \quad x \in \mathcal{H} .
$$

REMARK 5.2. Given $y \in \mathcal{H}$, an element $x_{0}$ satisfies (5) if and only if $\left\|A^{1 / 2}\left(y-B x_{0}\right)\right\| \leq\left\|A^{1 / 2}(y-B x)\right\|=\left\|A^{1 / 2}\left(y-B x_{0}\right)+A^{1 / 2} B\left(x_{0}-x\right)\right\|$ for all $x \in \mathcal{H}$, or equivalently $\left\langle A^{1 / 2} y-A^{1 / 2} B x_{0}, A^{1 / 2} B z\right\rangle=0$ for all $z \in \mathcal{H}$ (recall that given $a, b \in \mathcal{H}$, we have $\|a\| \leq\|a+t b\|$ for all $t \in \mathbb{C}$ if and only if $\langle a, b\rangle=0)$. Then $x_{0}$ is an $A$-LSS of $B x=y$ if and only if $x_{0}$ is a solution of

$$
B^{*} A B x=B^{*} A y .
$$

Equation (6) is the normal equation associated to (5).

The next two results generalize [7, Proposition 4.4] and [7, Lemma 4.6].

Proposition 5.3. Suppose $(A, R(B))$ is compatible and consider $y \in \mathcal{H}$, $y \neq 0$. Then $u \in \mathcal{H}$ is an $A$-LSS of $B x=y$ if and only if there exists $T \in \Pi(A, R(B))$ such that $B u=T y$.

Proof. Observe that $u \in \mathcal{H}$ is an $A$-LSS of $B x=y$ if and only if $\|B u-y\|_{A}=\inf _{\sigma \in R(B)}\|\sigma+y\|_{A}$, or $y-B u \in \operatorname{sp}\left(A^{1 / 2}, R(B), y\right)$; or equivalently, by Proposition 4.24, $B u=T y$ for some $T \in \Pi(A, R(B))$.

Corollary 5.4. Let $(A, N(B))$ be compatible and consider $x_{0} \in N(B)^{\perp}$, $x_{0} \neq 0$ and $u \in x_{0}+N(B)$. Then $\|u\|_{A} \leq\|x\|_{A}$ for all $x \in x_{0}+N(B)$ if and only if there exists $T \in \Pi(A, N(B))$ such that $u=(I-T) x_{0}$.

Proof. Since $x_{0} \in N(B)^{\perp}$ and $u \in x_{0}+N(B)$, we have $u=x_{0}+P_{N(B)} u$. Consider $T \in \Pi(A, N(B))$ such that $u=(I-T) x_{0}$, so that $P_{N(B)} u=-T x_{0}$. Since $x_{0} \neq 0$, by the previous proposition $u$ is an $A$-LSS of $P_{N(B)} x=-x_{0}$; hence $\left\|P_{N(B)} u+x_{0}\right\|_{A} \leq\left\|P_{N(B)} x+x_{0}\right\|_{A}$ for all $x \in \mathcal{H}$, or equivalently $\|u\|_{A} \leq\|x\|_{A}$ for all $x \in x_{0}+N(B)$. The converse follows by [7, Lemma 4.6].

The following concept was introduced by Rao and Mitra [24] for finitedimensional spaces.

Definition 5.5. An operator $G \in L(\mathcal{H})$ is called an $A$-inverse of $B$ if for each $y \in \mathcal{H}, G y$ is an $A$-LSS of $B x=y$, i.e.

$$
\|y-B G y\|_{A} \leq\|y-B x\|_{A}, \quad x \in \mathcal{H} .
$$

REMARK 5.6. If $G$ is an $A$-inverse of $B$ then $R(G)$ is not necessarily closed. In fact, if $A$ has infinite-dimensional nullspace, consider $G_{1} \in$ $L(N(A))$ such that $R\left(G_{1}\right)$ is not closed. It is easy to see that $G=G_{1} P_{N(A)}+$ $P_{N(A) \perp}$ is an $A$-inverse of $I$.

The following result gives a necessary and sufficient condition for an operator $B$ with closed range to admit an $A$-inverse. 
Proposition 5.7. The operator $B$ admits an $A$-inverse if and only if $(A, R(B))$ is compatible.

Proof. Let $G \in L(\mathcal{H})$ be an $A$-inverse of $B$ and consider $T=B G$. Then $R(T) \subseteq R(B)$ and $\|y-T y\|_{A}=\|y-B G y\|_{A} \leq\|y-B x\|_{A}$ for all $x \in \mathcal{H}$, so that $T$ is an $A$-projection into $R(B)$. Then $(A, R(B))$ is compatible by Proposition 4.14 .

Conversely, if $(A, R(B))$ is compatible, using again Proposition 4.14, let $T$ be an $A$-projection into $R(B)$. Since $R(T) \subseteq R(B)$, by Douglas' theorem there exists $G \in L(\mathcal{H})$ such that $T=B G$. Therefore,

$$
\|y-B G y\|_{A}=\|y-T y\|_{A} \leq\|y-B x\|_{A} \quad \text { for } x \in \mathcal{H},
$$

so that $G$ is an $A$-inverse of $B$.

REMARK 5.8. It follows from the above proof that, if $G$ is an $A$-inverse of $B$ then $T=B G$ is an $A$-projection into $R(B)$. Conversely, given $T$ an $A$-projection into $R(B)$, the solutions of $B X=T$ are $A$-inverses of $B$.

The next result gives necessary and sufficient conditions for an operator $G \in L(\mathcal{H})$ to be an $A$-inverse of $B$.

Proposition 5.9. An operator $G \in L(\mathcal{H})$ is an $A$-inverse of $B$ if and only if $B^{*} A B G=B^{*} A$.

Proof. Let $G \in L(\mathcal{H})$ be an $A$-inverse of $B$. By Remark 5.8, $T=B G$ is an $A$-projection into $R(B)$. Hence, by Proposition 4.5, it follows that $P_{R(B)} A T=P_{R(B)} A$ so that $B^{*} A B G=B^{*} A$.

Conversely, consider $T=B G$; then $B^{*} A T=B^{*} A$, or equivalently, $P_{R(B)} A T=P_{R(B)} A$. Therefore, by Proposition 4.5 $T=B G$ is an $A$ projection into $R(B)$. Finally, by Remark 5.8, $G$ is an $A$-inverse of $B$.

Corollary 5.10. If $(A, R(B))$ is compatible, then the set of $A$-inverses of $B$ is

$$
\left(B^{*} A B\right)^{\dagger} B^{*} A+L\left(\mathcal{H}, N\left(B^{*} A B\right)\right) .
$$

5.1. Restricted weighted inverses. Throughout this section, $\mathcal{M}$ is a closed subspace of $\mathcal{H}$ such that $B(\mathcal{M})$ is closed, or equivalently, since $B$ has closed range, $\mathcal{M}+N(B)$ is a closed subspace of $\mathcal{H}$.

Definition 5.11. An operator $G \in L(\mathcal{H})$ is called an $A$-inverse of $B$ restricted to $\mathcal{M}$ if $R(G) \subseteq \mathcal{M}$ and for each $y \in \mathcal{H}$,

$$
\|y-B G y\|_{A} \leq\|y-B x\|_{A}, \quad \forall x \in \mathcal{M} .
$$

The concept of $A$-inverses restricted to $\mathcal{M}$ was introduced by Rao and Mitra 24] for finite-dimensional spaces.

In what follows we show that the existence of an $A$-inverse of $B$ restricted to $\mathcal{M}$ is equivalent to the compatibility of the pair $(A, B(\mathcal{M}))$. 
Lemma 5.12. An operator $G \in L(\mathcal{H})$ is an $A$-inverse of $B$ restricted to $\mathcal{M}$ if and only if $R(G) \subseteq \mathcal{M}$ and $G$ is an $A$-inverse of $B P_{\mathcal{M}}$.

Proof. Straightforward.

Remark 5.13. By the previous lemma and Proposition 5.9 applied to $B P_{\mathcal{M}}$, we know that $G$ is an $A$-inverse of $B$ restricted to $\mathcal{M}$ if and only if $R(G) \subseteq \mathcal{M}$ and $P_{\mathcal{M}}\left(B^{*} A B G-B^{*} A\right)=0$.

Proposition 5.14. Suppose $(A, B(\mathcal{M}))$ is compatible and consider $T \in$ $\Pi(A, B(\mathcal{M}))$. Then the reduced solution of

$$
B P_{\mathcal{M}} X=T
$$

is an $A$-inverse of $B$ restricted to $\mathcal{M}$.

Proof. Let $G_{0}$ be the reduced solution of $B P_{\mathcal{M}} X=T$. Then $R\left(G_{0}\right) \subseteq$ $N\left(B P_{\mathcal{M}}\right)^{\perp}$. By Remark 5.8, $G_{0}$ is an $A$-inverse of $B P_{\mathcal{M}}$. Since $N\left(B P_{\mathcal{M}}\right)=$ $(\mathcal{M} \cap N(B)) \oplus \mathcal{M}^{\perp}$, we see that $R\left(G_{0}\right) \subseteq \mathcal{M}$. Therefore, by Lemma 5.12 , $G_{0}$ is an $A$-inverse of $B$ restricted to $\mathcal{M}$.

COROLlary 5.15. The operator $B$ admits an $A$-inverse restricted to $\mathcal{M}$ if and only if $(A, B(\mathcal{M}))$ is compatible.

Proof. If $G$ is an $A$-inverse of $B$ restricted to $\mathcal{M}$, then by Remark 5.12 , $G$ is an $A$-inverse of $B P_{\mathcal{M}}$, so that $(A, B(\mathcal{M}))$ is compatible (see Proposition 5.7). The converse follows by Proposition 5.14.

5.2. $A_{1} A_{2}$-inverses and weak weighted inverses. Throughout this section, $B \in L(\mathcal{H})$ is a closed range operator and $A_{1}, A_{2} \in L(\mathcal{H})^{+}$.

Definition 5.16. An operator $G \in L(\mathcal{H})$ is called an $A_{1} A_{2}$-inverse of $B$ if $G$ is an $A_{1}$-inverse of $B$ and, for each $y \in \mathcal{H}, G y$ has minimum $A_{2}$-seminorm among the $A_{1}$-LSS of $B x=y$.

In [21, 24], $A_{1} A_{2}$-inverses are called minimum seminorm semileast squares inverses in the context of finite-dimensional spaces.

The next two results are proved in [21], for finite-dimensional Hilbert spaces. Our proofs, which follow the same ideas, are included for the sake of completeness.

Proposition 5.17. An operator $G \in L(\mathcal{H})$ is an $A_{1} A_{2}$-inverse of $B$ if and only if

(i) $B^{*} A_{1} B G=B^{*} A_{1}$,

(ii) $R\left(A_{2} G\right) \subseteq N\left(A_{1} B\right)^{\perp}$.

Proof. By Proposition 5.9, $G$ is an $A_{1}$-inverse of $B$ if and only if $B^{*} A_{1} B G$ $=B^{*} A_{1}$. Let $G$ be an $A_{1}$-inverse of $B$. It remains to prove that $G y$ has minimum $A_{2}$-seminorm among the $A_{1}$-LSS of $B x=y$ for each $y \in \mathcal{H}$ if and 
only if $R\left(A_{2} G\right) \subseteq \overline{R\left(B^{*} A_{1}^{1 / 2}\right)}$. Observe that given $y \in \mathcal{H}$, by Remark 5.2 any $A_{1}$-LSS of $B x=y$ can be written as

$$
x_{0}=\tilde{x}+P_{N\left(B^{*} A_{1} B\right)} z,
$$

where $\tilde{x}=G y$ is a solution of (6) (i.e. $B^{*} A_{1} B \tilde{x}=B^{*} A_{1} y$ ) and $z \in \mathcal{H}$. Then

$$
\|G y\|_{A_{2}} \leq\left\|G y+P_{N\left(B^{*} A_{1} B\right)} z\right\|_{A_{2}} \quad \text { for all } z \in \mathcal{H}
$$

if and only if

$$
\left\|A_{2}^{1 / 2} G y\right\| \leq\left\|A_{2}^{1 / 2} G y+A_{2}^{1 / 2} P_{N\left(B^{*} A_{1} B\right)} z\right\| \quad \text { for all } z \in \mathcal{H},
$$

or equivalently $\left\langle A_{2} G y, P_{N\left(B^{*} A_{1} B\right)} z\right\rangle=0$ for all $z \in \mathcal{H}$, or $P_{N\left(B^{*} A_{1} B\right)} A_{2} G=$ 0 . Therefore, $G$ is an $A_{1} A_{2}$-inverse of $B$ if and only if $B^{*} A_{1} B G=B^{*} A_{1}$ and $R\left(A_{2} G\right) \subseteq N\left(B^{*} A_{1} B\right)^{\perp}=N\left(A_{1} B\right)^{\perp}$.

Proposition 5.18. If $G$ is an $A_{1} A_{2}$-inverse of $B$, then

(i) $A_{1} B G B=A_{1} B, \quad A_{1} B G=(B G)^{*} A_{1}$,

(ii) $A_{2} G B G=A_{2} G, \quad A_{2} G B=(G B)^{*} A_{2}$.

Proof. If $G$ is an $A_{1} A_{2}$-inverse of $B$ then $G$ is an $A_{1}$-inverse of $B$. Therefore, by Proposition 5.9. $B^{*} A_{1} B G=B^{*} A_{1}$, hence $A_{1} B G=(B G)^{*} A_{1} B G$ $\geq 0$, so that $B G$ is $A_{1}$-selfadjoint. Also, $A_{1} B=(B G)^{*} A_{1} B=A_{1} B G B$ and (i) holds. To prove (ii) observe that $R(I-G B) \subseteq N\left(B^{*} A_{1} B\right)$ because $B^{*} A_{1} B G B=B^{*} A_{1} B$. Therefore for each $y \in \mathcal{H}$, by Remark 5.2, it follows that

$$
x=G y+(I-G B) z, \quad z \in \mathcal{H},
$$

is a solution of the normal equation (6) and then it is an $A_{1}$-LSS of $B x=y$. Since $G$ is an $A_{1} A_{2}$-inverse of $B$, we have $\|G y\|_{A_{2}} \leq\|G y+(I-G B) z\|_{A_{2}}$ for all $z \in \mathcal{H}$, or equivalently $\left\|A_{2}^{1 / 2} G y\right\| \leq\left\|A_{2}^{1 / 2} G y+A_{2}^{1 / 2}(I-G B) z\right\|$ for all $z \in \mathcal{H}$, hence $\left\langle A_{2} G y,(I-G B) z\right\rangle=0$ for all $z \in \mathcal{H}$, or $G^{*} A_{2}(I-G B)=0$. Finally, in the same way as we did in (i), $G^{*} A_{2}=G^{*} A_{2} G B$ implies (ii) (actually, both conditions are equivalent).

Corollary 5.19. Suppose the pairs $\left(A_{1}, R(B)\right)$ and $\left(A_{2}, N\left(A_{1} B\right)\right)$ are compatible. Then

$$
G=\left(I-T_{2}\right) B^{\dagger} T_{1}
$$

is an $A_{1} A_{2}$-inverse of $B$ for every $T_{1} \in \Pi\left(A_{1}, R(B)\right)$ and for every $T_{2} \in$ $\Pi\left(A_{2}, N\left(A_{1} B\right)\right)$.

Proof. For $G$ as in the statement, we have

$$
A_{1} B G B=A_{1} B B^{\dagger} T_{1} B-A_{1} B T_{2} B^{\dagger} T_{1} B=A_{1} T_{1} B=A_{1} B,
$$

because $R\left(T_{1}\right) \subseteq R(B), R\left(T_{2}\right) \subseteq N\left(A_{1} B\right)$ and $A_{1} T_{1} P_{R(B)}=A_{1} P_{R(B)}$. Also, 
observe that

$$
\begin{aligned}
(B G)^{*} A_{1} & =\left(B B^{\dagger} T_{1}-B T_{2} B^{\dagger} T_{1}\right)^{*} A_{1}=T_{1}^{*} A_{1}-\left(A_{1} B T_{2} B^{\dagger} T_{1}\right)^{*} \\
& =A_{1} T_{1}=A_{1} B G .
\end{aligned}
$$

Finally, by Proposition 4.9 and Remark 4.18 .

$$
\begin{aligned}
R\left(A_{2} G\right) & \subseteq A_{2} R\left(I-T_{2}\right) \subseteq A_{2}\left[R\left(A_{2} T_{2}\right)^{\perp}\right]=A_{2}\left[\left(A_{2} N\left(A_{1} B\right)\right)^{\perp}\right] \\
& =A_{2}\left[A_{2}^{-1}\left(N\left(A_{1} B\right)^{\perp}\right)\right] \subseteq N\left(A_{1} B\right)^{\perp} .
\end{aligned}
$$

Therefore, Proposition 5.17 implies that $G$ is an $A_{1} A_{2}$-inverse of $B$.

Proposition 5.20. The operator $B$ admits an $A_{1} A_{2}$-inverse if and only if the pairs $\left(A_{1}, R(B)\right)$ and $\left(A_{2}, N\left(A_{1} B\right)\right)$ are compatible.

Proof. Suppose $B$ admits an $A_{1} A_{2}$-inverse. Then, by Proposition 5.7, the pair $\left(A_{1} R(B)\right)$ is compatible. The pair $\left(A_{2}, N\left(A_{1} B\right)\right)$ turns out to be compatible by [18, Proposition 3.9]. The converse follows by the previous result.

Definition 5.21. An operator $G \in L(\mathcal{H})$ is called a weak $A_{1} A_{2}$-inverse of $B$ if

$$
\begin{cases}A_{1} B G B=A_{1} B, & A_{1} B G=(B G)^{*} A_{1}, \\ A_{2} G B G=A_{2} G, & A_{2} G B=(G B)^{*} A_{2} .\end{cases}
$$

If $A_{1}=A_{2}=I$ and $G$ is a weak $A_{1} A_{2}$-inverse of $B$, then $G=B^{\dagger}$.

Observe that if $G \in L(\mathcal{H})$ is an $A_{1} A_{2}$-inverse of $B$ then, by Proposition 5.18, $G$ is a weak $A_{1} A_{2}$-inverse of $B$.

REMARK 5.22. Observe that (7) is equivalent to $B^{*} A_{1} B G=B^{*} A_{1}$ and $G^{*} A_{2} G B=G^{*} A_{2}$.

Lemma 5.23. An operator $G \in L(\mathcal{H})$ is a weak $A_{1} A_{2}$-inverse of $B$ if and only if $G$ is an $A_{1}$-inverse of $B$ and $B$ is an $A_{2}$-inverse of $G$.

Proof. Apply Remark 5.22 and Proposition 5.9.

In [7, the authors defined a weighted generalized inverse of $B$ to be any operator $C \in L(\mathcal{H})$ such that

(8) $\quad B C B=B, \quad C B C=C, \quad A_{1} B C=(B C)^{*} A_{1}, \quad A_{2} C B=(C B)^{*} A_{2}$. In [7, Theorem 3.1], it is proved that the pairs $\left(A_{1}, R(B)\right)$ and $\left(A_{2}, N(B)\right)$ are compatible if and only if $B$ admits a weighted generalized inverse. Observe that in this case, $C$ is a weak $A_{1} A_{2}$-inverse of $B$.

Also, $C$ is a weighted generalized inverse of $B$ if and only if $B C \in$ $\mathcal{P}(A, R(B))$ and $I-C B \in \mathcal{P}(A, N(B))$. In order to generalize this, we now consider the solutions of the system

$$
\left\{\begin{array}{l}
B G \in \Pi\left(A_{1}, R(B)\right), \\
I-G B \in \Pi\left(A_{2}, N(B)\right) .
\end{array}\right.
$$


Proposition 5.24. An operator $G \in L(\mathcal{H})$ is a solution of (9) if and only if

$$
B G B=B, \quad A_{1} B G=(B G)^{*} A_{1}, \quad A_{2} G B=(G B)^{*} A_{2} .
$$

Proof. Note that $G$ is a solution of $(9)$ if and only if $A_{1} B G=(B G)^{*} A_{1}$, $A_{1} B G P_{R(B)}=A_{1} P_{R(B)}, R(I-G B) \subseteq N(B), A_{2}(I-G B)=(I-G B)^{*} A_{2}$ and $A_{2}(I-G B) P_{N(B)}=A_{2} P_{N(B)}$. Equivalently, $A_{1} B G=(B G)^{*} A_{1}, A_{1} B G B$ $=A_{1} B, B(I-G B)=0, A_{2} G B=(G B)^{*} A_{2}$, or $B G B=B, A_{1} B G=$ $(B G)^{*} A_{1}$ and $A_{2} G B=(G B)^{*} A_{2}$.

COROLLARY 5.25. The following statements are equivalent:

(i) system (9) admits a solution,

(ii) the pairs $\left(A_{1}, R(B)\right)$ and $\left(A_{2}, N(B)\right)$ are compatible,

(iii) $B$ admits a weighted generalized inverse.

Proof. (i) $\Rightarrow$ (ii) is straightforward; (ii) $\Rightarrow$ (iii) follows by [7, Theorem 3.1]; and (iii) $\Rightarrow(\mathrm{i})$ is a consequence of the previous proposition.

By Proposition 5.24 and Corollary 5.25 it follows that (8) has a solution if and only if 10 has a solution.

\section{References}

[1] W. N. Anderson Jr. and G. E. Trapp, Shorted operators. II, SIAM J. Appl. Math. 28 (1975), 60-71.

[2] J. Antezana, G. Corach, M. Ruiz, and D. Stojanoff, Nullspaces and frames, J. Math. Anal. Appl. 309 (2005), 709-723.

[3] M. Atteia, Généralisation de la définition et des propriétés des «spline-fonctions », C. R. Acad. Sci. Paris 260 (1965), 3550-3553.

[4] S. R. Caradus, Generalized Inverses and Operator Theory, Queen's Papers in Pure Appl. Math. 50, Queen's Univ., Kingston, ON, 1978.

[5] G. Cassier and L. Suciu, Mapping theorems and similarity to contractions for classes of A-contractions, in: Hot Topics in Operator Theory, Theta Ser. Adv. Math. 9, Theta, Bucureşti, 2008, 39-58.

[6] G. Corach, J. I. Giribet, and A. Maestripieri, Sard's approximation processes and oblique projections, Studia Math. 194 (2009), 65-80.

[7] G. Corach and A. Maestripieri, Weighted generalized inverses, oblique projections, and least-squares problems, Numer. Funct. Anal. Optim. 26 (2005), 659-673.

[8] G. Corach, A. Maestripieri, and D. Stojanoff, Oblique projections and Schur complements, Acta Sci. Math. (Szeged) 67 (2001), 337-356.

[9] G. Corach, A. Maestripieri, and D. Stojanoff, Generalized Schur complements and oblique projections, Linear Algebra Appl. 341 (2002), 259-272.

[10] G. Corach, A. Maestripieri, and D. Stojanoff, Oblique projections and abstract splines, J. Approx. Theory 117 (2002), 189-206.

[11] G. Corach, A. Maestripieri, and D. Stojanoff, A classification of projectors, in: Topological Algebras, Their Applications, and Related Topics, Banach Center Publ. 67, Inst. Math., Polish Acad. Sci., 2005, 145-160. 
[12] G. Corach, A. Maestripieri, and D. Stojanoff, Projections in operator ranges, Proc. Amer. Math. Soc. 134 (2006), 765-778.

[13] R. G. Douglas, On majorization, factorization, and range inclusion of operators on Hilbert space, Proc. Amer. Math. Soc. 17 (1966), 413-415.

[14] Y. C. Eldar, Sampling with arbitrary sampling and reconstruction spaces and oblique dual frame vectors, J. Fourier Anal. Appl. 9 (2003), 77-96.

[15] Y. C. Eldar and T. Werther, General framework for consistent sampling in Hilbert spaces, Int. J. Wavelets Multiresolution Information Process. 3 (2005), 497-509.

[16] L. Eldén, Perturbation theory for the least squares problem with linear equality constraints, SIAM J. Numer. Anal. 17 (1980), 338-350.

[17] P. A. Fillmore and J. P. Williams, On operator ranges, Adv. Math. 7 (1971), 254-281.

[18] J. I. Giribet, A. Maestripieri, and F. Martínez Pería, A geometrical approach to indefinite least squares problems, Acta Appl. Math. 111 (2010), 65-81.

[19] S. Hassi and K. Nordström, On projections in a space with an indefinite metric, Linear Algebra Appl. 208/209 (1994), 401-417.

[20] A. Maestripieri and F. Martínez Pería, Decomposition of selfadjoint projections in Krein spaces, Acta Sci. Math. (Szeged) 72 (2006), 611-638.

[21] S. K. Mitra and C. R. Rao, Projections under seminorms and generalized Moore Penrose inverses, Linear Algebra Appl. 9 (1974), 155-167.

[22] M. Z. Nashed, On generalized inverses and operator ranges, in: Functional Analysis and Approximation, Birkhäuser, Basel, 1981, 85-96.

[23] M. Z. Nashed, Inner, outer, and generalized inverses in Banach and Hilbert spaces, Numer. Funct. Anal. Optim. 9 (1987), 261-325.

[24] C. R. Rao and S. K. Mitra, Theory and application of constrained inverse of matrices, SIAM J. Appl. Math. 24 (1973), 473-488.

[25] A. Sard, Approximation and variance, Trans. Amer. Math. Soc. 73 (1952), 428-446.

[26] L. Suciu, Ergodic properties for regular A-contractions, Integral Equations Operator Theory 56 (2006), 285-299.

[27] L. Suciu, Quasi-isometries in semi-Hilbertian spaces, Linear Algebra Appl. 430 (2009), 2474-2487.

[28] Y. Takane, Y. Tian and H. Yanai, On constrained generalized inverses of matrices and their properties, Ann. Inst. Statist. Math. 59 (2007), 807-820.

[29] Y. Tian and Y. Takane, On V-orthogonal projectors associated with a semi-norm, Ann. Inst. Statist. Math. 61 (2009), 517-530.

G. Corach, A. Maestripieri

Departamento de Matemática, FI-UBA

and

Instituto Argentino de Matemática

Buenos Aires, Argentina

E-mail: gcorach@fi.uba.ar

amaestri@fi.uba.ar
G. Fongi

Instituto Argentino de Matemática

Buenos Aires, Argentina

E-mail: gfongi@gmail.com

Received September 5, 2012

Revised version June 5, 2013 